\title{
Motivación, satisfacción laboral y estado de flow en los trabajadores de la salud
}

\author{
Pizarro Ruz, Jenny ${ }^{1}$ \\ Fredes Collarte, Dailet ${ }^{2}$ \\ Inostroza Peña, Carolina ${ }^{3}$ \\ Torreblanca Urbina. Elisa ${ }^{4}$
}

\section{Resumen}

Actualmente existe una mayor preocupación sobre el trabajo saludable en las organizaciones, lo que implica tanto la promoción del bienestar físico como psicológico, la mayoría de los estudios sobre bienestar se han centrado en evaluar, casi exclusivamente los aspectos negativos del trabajo.Como propósito esta investigación busca explorar desde la perspectiva de la psicología organizacional positiva la relación entre tres variables motivación laboral, satisfacción laboral y el estado de flow, en trabajadores de Centros de Salud Familiar (CESFAM) de la ciudad de lquique. El estado de Flow es una estado actual donde las personas se hayan involucradas en su actividad y nada más parece importarles, la actividad por sí misma es tan placentera que las personas la realizan aunque tengan un gran coste, por el puro motivo y placer de hacerla. De este estudio participaron 214 funcionarios, de salud primaria de la ciudad de Iquique, Chile, los que respondieron diferentes instrumentos relacionados con las variables en estudio. Esta investigación está basada en un diseño ex post facto correlacional mediante el uso de técnicas de correlación bivariada. Los resultados dan cuenta de la presencia positiva del estado de Flow dentro de la organización, y ha sido posible establecer la existencia de una relación directa y significativa entre las variables estudiadas.

Palabras clave: estado de flow; motivación; satisfacción laboral; trabajadores

Recibido: 20.02.19 Aceptado: 20.06.19

Psicóloga y Académica, Universidad de Tarapacá, Iquique Chile. Mg. Psicología del Trabajo las Organizaciones y los Recursos Humanos, Universidad Jaumei I, España. jem.0910@gmail.comcódigo ORCID: 0000-00021746-7008

2 Master en Dirección y Gestión de Recursos Humanos, Universidad Internacional de La Rioja, España. Académica, Universidad de Tarapacá, Iquique Chile. dfredesc@academicos.uta.cl ,: 0000-0002-5844-6975.

3 Psicóloga, Universidad de Tarapacá, Iquique, Chile. Pos- título en Mediación Familiar y Laboral. carolinainostroza49@gmail.com

4 Psicóloga, Universidad de Tarapacá, Iquique, Chile. Asesor externo Mutual de Seguridad. torreblancaelisa@ gmail.com 


\title{
Motivation and labor satisfaction and estate flow public health workers
}

\begin{abstract}
Currently there is a greater concern about healthy work in organizations, which implies both the promotion of physical and psychological wellbeing, most welfare studies have focused on evaluating almost exclusively the negative aspects of work. As a purpose this research seeks to explore from the perspective of positive organizational psychology the relationship between three variables Work Motivation, Labor Satisfaction and the state of Flow, in workers of Family Health Centers. The state of Flow is a current state where people have been involved in their activity and nothing else seems to matter, the activity itself is so pleasant that people do it even if they have a great cost, for the pure reason and pleasure of doing it. Of this study, 214 primary health officials from the city of lquique, Chile, participated, who answered different instruments related to the variables under study. This research is based on an ex post facto correlational design through the use of bivariate correlation techniques. The results show the positive presence of Flow's status within the organization, and it has been possible to establish the existence of a direct and significant relationship between the variables studied.
\end{abstract}

Keywords: Flow, motivation, job satisfaction, workers.

\section{Introducción}

La Organización Mundial de la salud ha definido el concepto de salud como: "Un estado de completo bienestar físico, mental y social y no solamente la ausencia de enfermedad o dolencia" (OMS, 2001). Sobre esta definición, en un análisis bibliométrico realizado a partir de las publicaciones de PsycINFO durante los años 2000 al 2008, las investigaciones desarrolladas en relación al bienestar mental representaron tan solo el $5.2 \%$ de la producción intelectual global de ese periodo, tal como señalaron Schui y Krampe (2010), el bienestar ha sido relativamente poco estudiado. Sin embargo, desde la aparición y auge de la Psicología Positiva, existe una tendencia al aumento de las investigaciones del bienestar mental (Millán, García-Álvarez,
D'Aubeterre, 2014).

En este sentido, la OMS (2013) planteó una estructura del plan de acción integral sobre salud mental, con la finalidad de fomentar el bienestar mental, poniendo en práctica, estrategias de promoción y prevención en el campo de la salud mental, así como también fortalecer los sistemas de información, los datos científicos y las investigaciones sobre la salud mental. No obstante, en nuestro país se ha observado que existe un considerable aumento de las enfermedades mentales cuyo origen es laboral (Ministerio de Salud, 2014) y por esta razón, los objetivos de salud proyectados al año 2020 plantean disminuir la incidencia de enfermedades profesionales, asociadas en muchas ocasiones a las exigencias laborales percibidas y otros factores psicosociales. 
En este sentido, es importante destacar que la salud y el trabajo son derechos humanos fundamentales, por lo que resulta inaceptable la pérdida de uno de ellos en el ejercicio del otro. El trabajo, en nuestra sociedad se relaciona de una forma directa con la calidad y expectativas de la vida de las personas. Considerando que desde hace años se conoce que las condiciones y medio ambiente de trabajo pueden ser fuente de enfermedad 0 salud (Santander y Tordesilla, 2005). Siendo un aspecto determinante para la salud de los trabajadores la relación entre la organización y el trabajador, particularmente la forma en que el trabajador percibe la relación, su trabajo y su entorno de trabajo.

Ahora bien, en esta relación se generan condiciones que son relevantes para la satisfacción, la motivación y el rendimiento laboral (Granados, Salazar y Gómez, 2011). En función de cómo se defina y se desarrolle la relación, se observarán consecuencias positivas o negativas en la salud laboral de los trabajadores (Ospina, García, Moliner, Molina, Martínez, 2012). Dado que los problemas de salud mental afectan a los entornos de trabajo, esta investigación se enfocará en estudiar factores positivos como el Flow, la Motivación Laboral y Satisfacción Laboral, en los Centros de Salud de Atención Primaria de la ciudad de Iquique, en los cuales se encuentran trabajadores como, médicos, dentistas, enfermeras, asistentes sociales, técnicos paramédicos, nutricionistas, matronas, auxiliares de servicio, administrativos, entre otros (Santander y Tordesilla, 2005). Debido a que en estas profesiones es más probable que se encuentren oportunidades de realización personal, centrándose la atención en el propio desarrollo y no sólo en los resultados, proporcionando mayor frecuencia de situaciones intrínsecamente gratificantes, que otros trabajos que se presentan más rutinarios y menos creativos.

Para Csikszentmihalyi (2013) la activación motivacional en una actividad no depende sólo de la novedad o interés intrínseco del trabajo que se realiza, sino de la correspondencia entre ésta y los recursos personales de los que dispone una persona para afrontar la situación. Si la persona está satisfecha con su trabajo responderá adecuadamente a las exigencias de éste; si, por el contrario, está insatisfecha no será capaz de realizar su labor con eficiencia y calidad. Conjuntamente la insatisfacción laboral se reflejaría en todas las esferas de la vida del trabajador incluido su bienestar y Salud Laboral (García, 2010).

Esta investigación está basada en un diseño ex post facto correlacional mediante el uso de técnicas de correlación bivariada. Las variables incluidas en esta investigación son, Flow, Motivación Laboral y Satisfacción laboral hipotizándose que: $\mathrm{H}_{1}$ : Flow y la Satisfacción laboral se relacionan entre sí; $\mathrm{H}_{2}$ : Flow y la Motivación laboral se relacionan entre sí. En este estudio participaron voluntariamente, 214 trabajadores pertenecientes al área de salud primaria que trabajan en Centros de Salud Familiar. Los instrumentos utilizados en esta oportunidad fueron: para evaluar Flow, se utilizó la traducción al castellano de la Escala del Estado de Flujo (FlowStateScale-FSS-2) de Jackson y Marsh (1996) revisada al español por García-Calvo, Jiménez, Santos-Rosa, Reinay Cervelló (2008). Para evaluar Motivación, se utilizó la Escala de Motivación Autopercibida en base a los planteamientos de Quijano y Navarro (1998). Y para evaluar 
Satisfacción Laboral se utilizó el Escala de Satisfacción Laboral (NTP 394) propuesta por Warr, Cook y Wall (WCW, 1979), traducida al español y adaptada por Pérez y Fidalgo (2007) la cual fue creada para evaluar la satisfacción intrínseca $(\mathrm{SI})$ y satisfacción extrínseca (SE), ambos medidos desde un enfoque de disposición actitudinal (Cuadra y Veloso, 2010).

\section{Psicologia organizacional positiva}

La psicología positiva se define, como el estudio científico de las fortalezas y virtudes humanas, las cuales permiten adoptar una perspectiva más abierta respecto al potencial humano que incluyen tanto sus motivaciones como sus capacidades (Sheldon y King, 2001). Permitiendo identificar los determinantes individuales y colectivos de las experiencias positivas, con el objeto de desarrollar intervenciones que permitan a los individuos y las organizaciones lograr satisfacción en la vida como también alcanzar un mayor potencial de desarrollo (Seligman y Csikszentmihalyi, 2000).

En este sentido, la psicología positiva proporciona un esquema integral para describir y entender en qué consiste una buena vida (Park y Peterson, 2009). Este campo se puede dividir en cuatro áreas relacionadas entre sí:

- Experiencias subjetivas positivas (felicidad, plenitud, fluir).

- Rasgos individuales positivos (fortalezas del carácter, talentos, intereses, valores).

- Relaciones interpersonales positivas (amistad, matrimonio, compañerismo).

- Instituciones positivas (familias, escuelas, negocios, comunidades).
Por consiguiente, las instituciones positivas posibilitan el desarrollo de relaciones positivas, facilitando el surgimiento de rasgos positivos $\mathrm{y}$, al mismo tiempo, construyen experiencias subjetivas positivas (Park y Peterson, 2003). De ahí que este estudio se interesa en indagar en más profundidad el estudio del bienestar en los trabajadores a través de la psicología organizacional positiva. Para Salanova (2008) el concepto de Psicología Organizacional Positiva se entiende como el estudio científico del funcionamiento óptimo de las personas y de los grupos en las organizaciones, así como su gestión efectiva la cual ve al trabajador como un potencial motivador, y como un agente de distribución y aprovechamiento de los recursos laborales.

Esta área tiene su origen en las investigaciones realizadas por los psicólogos Martin Seligman y Mihaly Csikszentmihalyi, quienes se enfocaron en estudiar cómo se logra el óptimo desarrollo humano asociándolo al desarrollo de la creatividad y el sentido de flow en las labores rutinarias, de manera tal que se incremente el sentido de felicidad por medio del disfrute, de la labor cotidiana y potenciándose simultáneamente el desempeño laboral (Forbes, 2013).

De acuerdo con esta postura, se busca que las organizaciones puedan no sólo captar y retener a sus empleados, sino también la posibilidad de nutrirlos para que puedan sentirse satisfechos, productivos como también desarrollar su creatividad, con el fin de estar comprometidos con la organización, de esa manera lograr que la organización llegue a un estado flow a partir del cual se puedan lograr resultados extraordinarios (Nader, Peña y Santa-Bárbara, 2014). 


\section{FLOW: algunas reflexiones}

\author{
El flow es un constructo psicológico
} relacionado desde sus orígenes con los estudios sobre motivación intrínseca, introducido a principios de los setenta en la literatura científica por el psicólogo cognitivista social, Mihaly Csikszentmihalyi (Orta y Sicilia, 2015). A partir de su preocupación por los aspectos creativos, cognitivos y motivacionales de las personas, Csikszentmihalyi, en su obra "Flow": The Psychology of optimal Experience, establece que el mismo "estado espiritual extraordinario" que experimentan los artistas cuando crean su obra, puede encontrarse en otras personas que trabajan en actividades comunes (Salanova, Martínez, Cifré y Schaufeli, 2005).

El nombre de este concepto surge a partir de las descripciones dadas por algunas personas entrevistadas, que lo usaban como metáfora para describir sus sentimientos cuando estaban inmersos en actividades creativas (ajedrez, escalada, pintura, juego, baile), las cuales, en sí mismas, resultaban ser gratificantes y satisfactorias (Orta y Sicilia, 2015). Csíkszentmihályi define el Flow como el "estado actual donde las personas se hayan involucradas en la actividad y nada más parece importarles, la actividad por sí misma es tan placentera que las personas la realizan aunque tengan un gran coste, por el puro motivo de hacerla. También el Flow debería de ser una experiencia óptima, uno de los mejores estados, si no el mejor, o por lo menos estar a la par de esas recompensas homeostáticas que denominamos placer" (Csikszentmihalyi, 2013).

Es importante hacer hincapié en la distinción de los conceptos de "placer y disfrute". Hace esta diferenciación, ya que el concepto de Flow se relaciona directamente con el disfrute, el cual suele confundirse comúnmente con el concepto de placer. El placer es un sentimiento de satisfacción que se logra cuando la información en la conciencia nos dice que hemos conseguido cumplir con las expectativas controladas por los programas o por el condicionamiento social, siendo un componente importante de la calidad de vida, pero por sí mismo, no trae la felicidad. En cambio, el disfrute está caracterizado por este movimiento hacia delante: por un sentimiento de novedad, de realización. A diferencia del placer, el disfrute se caracteriza en las personas que pueden mantener una satisfacción duradera y esto a su vez, empuja hacia el crecimiento personal (Csikszentmihalyi, 2013).

Entonces Flow se experimenta cuando una persona está motivada y capacitada para realizar una actividad, se siente desafiado por la tarea, está totalmente concentrado hasta el punto de perder la noción temporal y emplea todas sus potencialidades, por lo tanto la persona se esfuerza al máximo sin darse cuenta y este esfuerzo está dirigido a la consecución de metas al tiempo que experimenta control sobre la situación y sobre sí mismo (Csikszentmihalyi, 2013). Este estado fue corroborado en una gran variedad de contextos, incluido el trabajo, la escuela, el tiempo libre y los deportes (Mesurado, 2008) experimentándose por todas las personas independientemente de la edad, sexo, cultura y situación económica, en resumen, se trata de un fenómeno universal, aun cuando pueden hacerse cosas muy diferentes (debido a la influencia cultural) para alcanzar una experiencia óptima (Csikszentmihalyi, 2013).

Ahora bien, Salanova et al, (2006) señalan que los avances en el estudio 
de Flow se han centrado en explorar los elementos y condiciones que hacen posible experimentarlo en los trabajadores, de modo que, si en una organización los empleados disfrutan con sus trabajos estando concentrados y absortos en la tarea que realizan, se puede decir que tienen experiencias positivas en su trabajo y posiblemente, podrían experimentar frecuentemente Flow. No obstante, aún no existen protocolos de actuación prefijados para el fomento de experiencias de Flow en organizaciones (Rodríguez, Cifre, Salanova y Aborg, 2008). Por otra parte, Rodríguez, et al, (2008) mencionan que "si se conocen las variables que componen la experiencia de flow en sí misma, y se consiguen concretar sus antecedentes y consecuentes, se podrán desarrollar métodos y herramientas para su diagnóstico y potenciación en el lugar de trabajo". La Dirección de cualquier empresa que quiera construir una organización donde los empleados estén motivados para contribuir y permanecer en ella según Csikszentmihalyi (2003) tiene tres opciones principales:

- Intentar que las condiciones objetivas del lugar del trabajo sean tan atractivas como sea posible como por ejemplo que conste con buena iluminación, que sea un lugar limpio y seguro, como también una paga justa, etc.

- Encontrar maneras de dar significado y valor añadido al trabajo realizado (variedad de tareas, autonomía, retos, puestos que se ajusten a la formación de los empleados).

- Seleccionar y recompensar a aquellos trabajadores que encuentren satisfacción en su trabajo.

Estos tres aspectos que indica
Csikszentmihalyi podrían ser un principio para propiciar los entornos saludables de trabajo que cualquier organización desea obtener. Implementando todas o algunas de estas estrategias se podría optimizar la calidad de vida laboral y, del mismo modo, optimizar la calidad de la organización (Rodríguez, et al, 2008).

En este orden de ideas, gran cantidad de autores han señalado que una persona que se siente feliz, funciona de manera más apropiada, vive mayor cantidad de años tiene mejor salud física y rinde mejor en el trabajo (Seligman y Csikszentmihalyi, 2000; Lyubomirsky, King y Diener, 2005; Diener y BiswasDiener, 2008; Castro, 2009, 2010).

Csikszentmihalyi (2013), propone los elementos que componen el Flow, y de esta manera entender mejor que hace tan gratificante las actividades agradables. $Y$ así lograr convertir los momentos más monótonos de la vida cotidiana (como el trabajo) en acontecimientos que ayuden a nuestro crecimiento personal.

Esa noción de meta alcanzable, junto con los aspectos aquí mencionados de interés, atención y "no percepción del paso del tiempo" nos llevan a pensar necesariamente en el Modelo de Reto Óptimo del psicólogo Mihaly Csikszentmihalyi (2013), que conduce al sujeto a la llamada "Experiencia de Flow'", o sea, a un estado en el cual las personas se hallan tan involucradas en la tarea que nada les parece más importante constituyendo una experiencia intrínsecamente placentera, que las personas intentarán repetir, siempre que sea posible, con el fin de volver a experimentar esa sensación.

\section{Modelo del reto}

Como hemos visto, la experiencia 
de Flow es un estado de disfrute, control y atención focalizada que se logra cuando las oportunidades de acción percibidas en el entorno emplean plenamente las capacidades de acción; es decir, cuando coinciden los desafíos y las habilidades, subjetivamente percibidas (Mesurado, 2008).

Es por esto, que el estado de Flow puede ser representado como un canal sobre un esquema de desafío versus habilidad, separando los estados de aburrimiento y ansiedad. Por lo tanto, la calidad de la experiencia es dinámica: si el desafío de la tarea disminuye, esta podría convertirse en algo aburrido y por otra parte si el desafío aumenta y las habilidades de uno no mejoran para hacer frente al desafío, entonces se experimenta un estado de ansiedad (Pearce, Ainley y Howard, 2005) (figura 1).

\section{Figura 1 \\ Modelo original de Flow de Csikszentmihalyi}

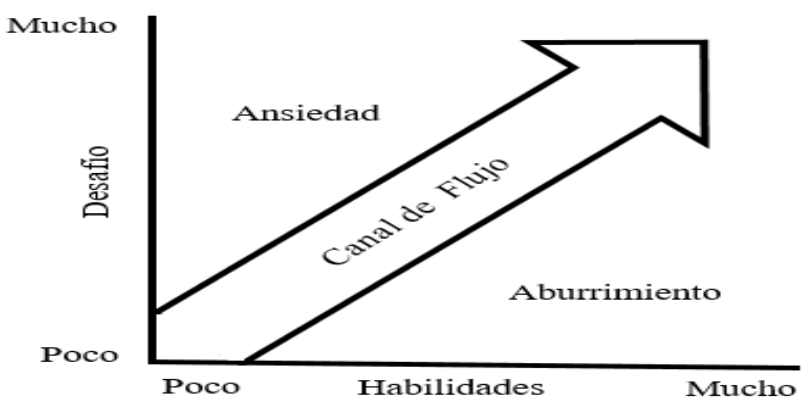

Fuente: Csikszentmihalyi, 2013).

En este sentido, Csíkszentmihályi se refiere al Flow como una experiencia autotélica acompañada de un estado de conciencia superior, que surge cuando los desafíos y habilidades se perciben por encima de un nivel medio en una situación de equilibrio entre ambos, Jackson y Csíkszentmihályi, llegan afirmar que esta relación de equilibrio entre desafíos y habilidades constituye la "regla de oro" (Csíkszentmihályi, 2013).

Ahora bien, es importante aclarar que la mayoría de las cosas que se realizan no son puramente autotélicas ni puramente exotélicas (es como se denomina a las actividades llevadas a cabo solo por razones externas), sino que son una combinación de las dos (Csikszentmihalyi, 2013).

En otras palabras, el flujo supone el equilibro entre los retos y las habilidades como por ejemplo, una buena actividad en el trabajo es la que presenta una gama muy alta de oportunidades para mejorar, permitiendo que los trabajadores experimenten 
como resultado (Csikszentmihalyi, 2013): Creatividad como deleite que siente al realizar el trabajo, desarrollo de habilidades, productividad y alta Autoestima.

En suma, en la medida que se suele sentir la necesidad de superación, que haya variedad en la tarea, así como también mayores posibilidades de aprender y de usar al máximo las facultades en el trabajo, se producirá el Flow. Este tipo de experiencias son comunes, por ejemplo, entre los trabajadores que tienen mayor nivel de compromiso, involucramiento, motivación y satisfacción en o por su trabajo (Csikszentmihalyi, 2013).

Así bien, es importante también estudiar las variables motivación laboral y satisfacción laboral en conjunto con el Flow ya que como lo plantea Zubiri (2013) la Motivación y la Satisfacción Laboral adquieren una relevancia significativa, debido a que son unas de las variables más estudiadas por el impacto que generan en la vida laboral y son un elemento fundamental para el éxito organizacional.

\section{Motivación laboral}

Tradicionalmente la motivación en el trabajo ha constituido un proceso de medular importancia tanto desde la perspectiva de la gestión organizacional como desde la perspectiva de la investigación (Steers, Mowday y Shapiro, 2004). Resulta significativo el número de teorías que se han desarrollado al respecto y la gran cantidad de investigaciones que se han llevado a cabo hasta el momento.

La motivación laboral hace referencia a la fuerza psicológica que impulsa a las personas a iniciar, mantener y mejorar sus tareas laborales (López,
2005). Es por esto que la motivación en el trabajo ha sido uno de los constructos más estudiados por la psicología del trabajo y de las organizaciones.

Quijano y Navarro (1998), sostienen que una visión más globalizadora e integradora de la motivación, es decir un modelo que integre los diversos aspectos y conceptos relevantes mejora la comprensión de la motivación laboral. En concreto el modelo integrado de Quijano y Navarro sobre la motivación en el trabajo se sustenta en: 1) los diferentes tipos de necesidades que puedan estar operando en las personas que las conducen a tener una conducta motivada, 2) la percepción de instrumentalidad o percepción de conexión entre el trabajo bien hecho y la satisfacción de necesidades, 3) la presencia e intervención de una serie de procesos cognitivos, tales como la percepción de autoeficacia, la percepción de equidad, el significado percibido del trabajo que se realiza, la conciencia sobre los resultados obtenidos y sobre la responsabilidad que se tiene sobre los mismos. (Carbón y Varela, 2011).

La motivación es un elemento que necesitan todos los individuos para la consecución de objetivos en cualquier aspecto de la vida. En el ámbito laboral, se necesitan factores o herramientas que motiven a las personas a realizar un trabajo satisfactorio, para que las organizaciones tengan éxito, ya que muchas veces los objetivos de la empresa solo se pueden conseguir con la voluntad de los trabajadores (Hoyal, 2015).

Para Csikszentmihalyi (2013) la activación motivacional no depende sólo de la novedad o interés intrínseco del trabajo en cuestión, sino de la correspondencia entre ésta y los recursos personales de los que se dispone para 
afrontar la situación en un equilibrio entre la competencia del individuo y el desafío implicado en la tarea, esto implica que si los trabajadores se encuentran más activos y motivados son los que tienen mayor probabilidad de progresar en el ámbito laboral, y además aquellos que han cambiado de estatus se esfuerzan más en el trabajo y desarrollan más conductas de innovación (Ripoll, Caballer, Martínez, García y Peiró, 1988). Como consecuencia dentro de las organizaciones, la motivación de los trabajadores que la conforman es fundamental para obtener beneficios y buenos resultados. (Walter, Gallegos y Velarde, 2013)

\section{Flow, motivación y satisfacción laboral}

Csikszentmihalyi durante sus estudios doctorales, en 1968, investigó sobre la Motivación intrínseca en un grupo de artistas, este interés surgió a partir de querer descubrir que era lo que los movía a pintar o esculpir con tanta concentración sin que nada pudiera distraerlos y sin que existiera una recompensa extrínseca de peso, como podría ser, obtener fama, éxito o bien una gratificación económica por la tarea realizada, de ahí que la investigación y la teoría sobre el Flow han tenido su origen en el deseo de entender este fenómeno de actividad motivada intrínsecamente. Es por esto que los antecedentes del Flow podemos situarlos en la teoría de la motivación, formulada en la década de los cincuenta y sesenta. Pero ha sido recientemente cuando el concepto ha adquirido entidad propia (Salanova et al., 2005).

Sin embargo, como lo señala Fullagar y Mills (2008), "la diferencia entre la experiencia de Flow y la
Motivación, está en que la primera centra su foco en la experiencia subjetiva (tanto afectiva como cognitiva) implicada durante la realización de una actividad, mientras que la motivación se focaliza en el motivo por el cual se realizó o se planea realizar una actividad".

La satisfacción laboral, es el tópico de carácter positivo más investigado en la historia de la Psicología Industrial y Organizacional; además, ocupa un papel central en muchas teorías, modelos sobre las actitudes y conductas de los individuos, debido a sus aplicaciones prácticas en la mejora de las vidas de los trabajadores y en la búsqueda de la "felicidad" y en el logro de la eficiencia organizacional (Pecino, Mañas, Díaz, López y Llopis, 2015).

Satisfacción Laboral se define como la actitud del trabajador frente a su propio trabajo, dicha actitud está basada en las creencias y valores que el trabajador desarrolla de su propio trabajo. Estas actitudes son determinadas conjuntamente por las características actuales del puesto como por las percepciones que tiene el trabajador de lo que debería ser (Feris y Castro, 2006). Asi como también Csikszentmihalyi (2013), precisa la Satisfacción Laboral como un estado emocional positivo y placentero de la percepción subjetiva de las experiencias laborales del sujeto.

En la investigación organizacional, Adams y Bond (2000) consideran la Satisfacción como una actitud circunscrita al ámbito laboral, siendo definida como el grado de afecto positivo hacia el trabajo o sus componentes. Asimismo Price y Mueller (1986) la definen "como el grado en el que a los individuos les gusta su trabajo" (Citado Figueiredo, Alberola, Gil-Monte y GarcíaJuesas, 2012). 
En este sentido el trabajador tendrá la satisfacción de sentirse apto para la tarea, conllevándolo a la permanencia en el trabajo y a la productividad laboral. Además, la Satisfacción Laboral resulta ser un indicador de bienestar psicológico (Mañas, Salvador, Boada, González y Agulló, 2007).

En la literatura mencionada, se ha propuesto que las experiencias que producen Flow y Satisfacción no se encuentran en el resultado, sino en el proceso de la actividad en su conjunto, lo cual permite una sensación más prolongada. Este fenómeno depende, por un lado, de la predisposición de los individuos a alcanzar una experiencia óptima $y$, por otro, de las influencias ambientales y quizás de las características de la actividad en sí misma.

Según los estudios de Csikszentmihalyi, concluyó que cuando se está en Flow, la actividad produce un estado mental tan satisfactorio que la persona la realiza sin importar la recompensa externa, aunque incluso requiera invertir un alto costo de energía en esta, o bien realizar un gran esfuerzo para alcanzar la meta (Smith, 2005).

Con el objeto de introducir la relación del flow con la satisfacción laboral es importante destacar que las características de las experiencias óptimas son comunes entre los trabajadores que tienen mayor nivel de compromiso, involucramiento, motivación y satisfacción en o por su trabajo.

Es decir, la característica más distintiva del Flow es quizá ésta: que es un "proceso" o "estado dinámico" y no un estado completamente estático adimensional. Esto impone la obligatoriedad de interacción entre sus diferentes componentes, lo que supone una compleja experiencia, capaz de producir placer por sí misma y no tanto por sus resultados (Fernández, Martín, Pérez y González, 2011). En palabras de Salanova: "La satisfacción no se encuentra en los resultados, sino en el proceso de la actividad en su conjunto, lo cual permite una sensación más prolongada" (Salanova, Martínez, Cifre y Schaufeli, 2005).

$\mathrm{Si}$ se repasa la literatura especializada, se encontrará que existen algunos trabajos que han conseguido demostrar que el alcanzar Flow se encuentra positivamente asociado con un sentido de plenitud y de satisfacción con la vida, (Asakawa, 2004, 2009; Asakawa y Yana, 2010; Han, 1988; Ishimura y Kodama, 2006; Peterson, Park y Seligman, 2005). En un estudio sobre el Flow realizado en la universidad de Chicago llamado "Optimal Experience in Work and Leisure" se basó en un método de muestreo para medir el Flow en contextos laborales dando cuenta que cuando las personas estaban en Flow, describían su trabajo o tiempo libre como una experiencia mucho más positiva que cuando no estaban en Flow, refiriéndose a la experiencia, como un estado que produce creatividad y satisfacción (Csikszentmihalyi y LeFevre, 1989).

\section{Flow y su relación con la motivación y la satisfacción laboral}

Esta investigación está basada en un diseño ex post facto correlacional mediante el uso de técnicas de correlación bivariada. Las variables incluidas en esta investigación son, Flow, Motivación Laboral y Satisfacción laboral hipottizandose que: $\mathrm{H}_{1}$ : Flow y la Satisfacción laboral se relacionan 
entre sí; $\mathrm{H}_{2}$ : Flow y la Motivación laboral se relacionan entre sí. Los resultados y análisis estadísticos se obtuvieron mediante la utilización del programa estadístico SPSS versión 23.0.

Un total de 214 funcionarios pertenecientes al área de salud primaria que trabajan en Centros de Salud Familiar participaron de manera voluntaria en el estudio. Del total 57 de ellos eran hombres $(26,89 \%)$ y 155 mujeres $(73,11 \%)$, con un promedio de edad de 37 años. Con el fin de acceder a los participantes se solicitaron las autorizaciones institucionales y consentimientos informados para la aplicación de los instrumentos requeridos, los que fueron aplicados según la disponibilidad horaria.

Los instrumentos utilizados en esta oportunidad y ya utilizados en estudios anteriores fueron: para evaluar Flow, se utilizó la traducción al castellano de la Escala del Estado de Flujo (FlowStateScale-FSS-2) de Jackson y Marsh (1996) revisada al español por García-Calvo, Jiménez, Santos-Rosa, Reinay Cervelló (2008). Esta versión del instrumento mide la disposición a experimentar el estado de Flow, consta de nueve dimensiones la cuales son: Balance entre la capacidad y nivel de desafío, fusión de la acción y la conciencia, metas claras, retroalimentación inmediata, concentración de la tarea a ejecutar, sensación de control, perdida de la conciencia de sí o inhibición, distorsión del sentido del tiempo y experiencia autotélica, las cuales se encuentran distribuidas en 36 ítems. Las respuestas a dicho cuestionario son cerradas y se responden con una escala tipo Likert cuya puntuación oscila entre: Muy desacuerdo $=1$, Desacuerdo $=2$, $\mathrm{Ni}$ acuerdo ni desacuerdo $=3, \mathrm{De}$ acuerdo=4, Muy de acuerdo=5.

La Adaptación española de la Escala del Estado de Flujo, cuenta con una fiabilidad de .70 resultando consistente entre la medición de sus factores de un $50 \%$. En esta muestra se evidencia una fiabilidad del .93 y posee una varianza explicada del $70 \%$ en sus nueve factores.

Para evaluar Motivación, se utilizó la Escala de Motivación Autopercibida en base a los planteamientos de Quijano y Navarro (1998). Es una escala de cuatro preguntas que indagan la motivación autopercibida de los trabajadores valorada en una escala Likert cuya puntuación oscila entre: Muy desacuerdo $=1$, Desacuerdo $=2$, $\mathrm{Ni}$ acuerdo $\mathrm{ni}$ desacuerdo $=3, \mathrm{De}$ acuerdo=4, Muy de acuerdo=5. Este instrumento cuenta con una fiabilidad de .67 y una varianza explicada en un $52.3 \%$ por un factor. Para esta muestra la fiabilidad es de un .79 y posee una varianza explicada de un $63,8 \%$ en un factor.

Para evaluar Satisfacción Laboral se utilizó el Escala de Satisfacción Laboral (NTP 394) propuesta por Warr, Cook y Wall (WCW, 1979), traducida al español y adaptada por Pérez y Fidalgo (2007) la cual fue creada para evaluar la satisfacción intrínseca (SI) y satisfacción extrínseca (SE), ambos medidos desde un enfoque de disposición actitudinal (Cuadra y Veloso, 2010). Esta escala está compuesta por dos dimensiones, satisfacción intrínseca y extrínseca, las cuales se encuentran distribuidas en 15 ítems que deben ser valorados en una escala Likert que va desde uno ("muy insatisfecho") a cinco ("muy satisfecho"). Su fiabilidad, estimada mediante el indicador Alfa de Cronbach, es de .88. Su estructura factorial evidenció un factor de primer orden que explicó el $72 \%$ de 
la variabilidad de los datos (Cuadra y Veloso, 2007). Para esta muestra la fiabilidad fue de un .91 y evidencio dos factores que explicaron el $53.6 \%$ de la varianza total.

En la tabla 1, se muestran las medias de cada variable las cuales se obtuvieron de la suma total de reactivos en la que se puede observar que los resultados obtenidos indican que hay presencia de las tres variables en los trabajadores de los centros de salud familiar.

Tabla 1

Estadísticos descriptivos

\begin{tabular}{lccl}
\hline Estadísticos descriptivos & & & \\
\hline & Media & Des.estánd. & $\mathrm{N}$ \\
\hline Flow & 144,790 & 19,895 & 214 \\
\cline { 1 - 1 } Satisfacción laboral & 57,981 & 11,321 & 214 \\
\cline { 1 - 1 } Motivación laboral & 18,299 & 2,244 & 214 \\
\hline
\end{tabular}

Fuente: elaboración propia

La tabla 2 contiene los coeficientes de correlación entre las variables Flow, Satisfacción Laboral, Motivación Laboral. Los resultados exponen que Flow presenta una correlación directamente proporcional y estadísticamente significativa con Motivación Laboral ( $r=, 519$; Sig.=,000). Además existe un $26.93 \%$ de la varianza del Flow que se explica con la relación que tiene con la Motivación Laboral.

Así mismo, se observa que se presenta una correlación directamente proporcional y estadísticamente significativa entre Flow y Satisfacción Laboral $(r=, 363$; Sig. $=, 000)$, lo cual significa que la Satisfacción Laboral también se relaciona con la experiencia de Flow. Además, existe un $13.18 \%$ de la varianza del Flow que se explica con la relación que tiene con Satisfacción Laboral. En síntesis, se puede observar que todas las variables del estudio se correlacionan significativamente al $99 \%($ sig. $=, 001)$.

\section{Tabla 2}

\section{Correlaciones entre las variables en estudio, Flow- Motivación y} Flow- Satisfacción

\begin{tabular}{llccc}
\hline & & Flow & Satis. Lab. & Motiv. Lab. \\
\hline \multirow{3}{*}{ Flow } & Correlación de Pear- & 1 &, $363^{* *}$ &, $519^{* *}$ \\
& son & &, 000 &, 000 \\
& Sig. (bilateral) & & 214 & 214 \\
\hline
\end{tabular}




\section{Cont... Tabla 2}

\begin{tabular}{llccc}
\hline & Correlación de Pear- &, $363^{* *}$ & 1 &, $479^{* *}$ \\
$\begin{array}{llc}\text { Satisfacción } \\
\text { laboral }\end{array}$ & Son &, 000 & &, 000 \\
& Sig. (bilateral) & 214 & 214 & 214 \\
& $\mathrm{~N}$ &, $519^{* *}$ &, $479^{* *}$ & 1 \\
& Correlación de Pear- &, 000 &, 000 & \\
$\begin{array}{l}\text { Motivación } \\
\text { laboral }\end{array}$ & Son & 214 & 214 & 214 \\
& Sig. (bilateral) & $\mathrm{N}$ & \\
\hline
\end{tabular}

Fuente: elaboración propia

De acuerdo a lo expuesto recientemente, es importante señalar que la correlación existente entre Flow y Motivación Laboral, se corrobora en los resultados de un estudio realizado en la Universidad de Argentina, el año 2015 llamado Motivación y Flow en deportistas, el cual tuvo por objetivo analizar la asociación existente entre la Motivación y el Flow en 120 jóvenes practicantes del deporte, si bien, esta investigación se realizó bajo un contexto deportivo los resultados obtenidos son similares a los de esta investigación, encontrándose una asociación positiva entre el Flow y la Motivación en los deportistas, además de poseer una alta disposición a las experiencias de Flow y una predominancia a la Motivación.

Así mismo, la correlación existente entre la variable Flow y Satisfacción Laboral condicen con los resultados de un estudio realizado en la universidad de Chicago, nombrado "Optimal Experience in Work and Leisure" sobre Flow, el cual se basó en un método de muestreo para medir la calidad el Flow en contextos laborales y de ocio, dando cuenta que cuando las personas estaban en Flow, describían su trabajo o tiempo libre como una experiencia mucho más positiva que cuando no estaban en Flow, refiriéndose a la experiencia, como un estado que produce creatividad y satisfacción. (Csikszentmihalyi y LeFevre, 1989). A partir de esto, es posible aseverar mediante los resultados de este estudio, que los trabajadores de la Salud Primaria de Iquique no solo experimentan estado de Flow, sino que también Motivación Laboral y Satisfacción laboral las cuales al mismo tiempo se relacionan directamente proporcional y positivamente con las experiencias de Flow, lo que conllevaría a tener buena salud mental en los trabajadores y permite mejorar aspectos de la organización.

\section{Conclusiones}

La presencia de Flow, Motivación Laboral y Satisfacción Laboral en los trabajadores de la Salud Primaria, precisan una alta presencia de factores positivos. Entendiendo que los aspectos de una organización son relevantes en la vida del trabajador es importante recalcar que según Csikszentmihalyi una buena actividad en el trabajo es la que presenta una gama muy alta de oportunidades para mejorar, permitiendo que los trabajadores experimenten como resultado; Creatividad como deleite que 
siente al realizar su trabajo, ejecución máxima, es decir los trabajadores que dan su mejor esfuerzo, experimentan por lo general la sensación de flujo y por ende progresan de manera más eficaz en dicha actividad, desarrollando distintas habilidades, así como, la capacidad de potenciar ciertas destrezas que el trabajador ya posee. En consecuencia, cuando se experimenta sensación de Flow los trabajadores manifiestan estar más satisfechos y más motivados.

Es importante señalar limitaciones que deben ser tenidas en cuenta en futuras nuevas aproximaciones, por ejemplo, no haber tomado en cuenta factores como el nivel educativo y la ocupación del trabajador. Lo que podría haber enriquecido el estudio en sí, debido a que los resultados hubiesen sido más precisos. Como lo plantea Csikszentmihalyi (1990) la posibilidad de encontrar experiencias de Flow en todas las profesiones, no parece tener que ver con la edad ni con el género, sino más bien con el nivel educativo y con el tipo de ocupación que se realiza. Así pues, son más variables relacionadas con la educación y el trabajo, y no tanto demográficas las que se relacionan con el estado Flow. Siendo las personas con mayor nivel educativo las que desempeñan ocupaciones más recompensantes intrínsecamente, como directivos y supervisores ya que son los que puntúan más alto en las dimensiones del Flow.

\section{Referencias bibliográficas}

Adams A, Bond S (2000), Hospital nurses job satisfaction, individual and organizational characteristics. Journal of Advanced Nursing, 32(3), 536543.

Asakawa K (2004), Flow experience and autotelic personality in Japanese college students: How do they experience challenges in daily life? Journal of Happiness Studies, 5, 123-154.

Asakawa K, Yana K (2010), Applying Flow Theory to the Evaluation of the Quality of experience in a Summer School Program Involving E-interaction. Hosei University Repository.

Bounous B, Carbón G, Varela L (2011), Estructura del Puesto de Trabajo y Motivación Laboral. Universidad de Uruguay, 2011, P 21.

Castro A (2009), El bienestar psicológico: Cuatro décadas de progreso. Revista Interuniversitaria de Formación del Profesorado, 66(23, 3), 43-72.

Castro A (2010), Fundamentos de psicología positiva. Buenos Aires: Paidós.

Combombo L, Zito M, Cortese C (2013), Experiences and tools.

Contreras F, Esquerra G (2006), Psicología positiva: una nueva perspectiva en psicología.

Cuadra A, Flores I, Gajardo E (2001), Diagnóstico organizacional de las políticas de recursos humanos y su relación con la motivación y satisfacción laboral en los funcionarios del Área Académica del Centro de Formación Técnica de Tarapacá, en las localidades de Putre, Arica e Iquique (C.F.T.), Facultad de Ciencias Sociales y Jurídicas, Universidad de Tarapacá, Arica.

Cuadra A, Veloso C (2007), Liderazgo, clima y satisfacción laboral en las organizaciones. Revista de Humanidades y Ciencias Sociales, 2(22), 43-58.

Csikszentmihalyi M (1990), Finding Flow: The psychology of optimal experience. New York, NY: Harrer 
and Row.

Csíkszentmihályi M (1997), Finding flow: The Psychology of Engagement with Everyday LifeNewYork: Harper-Collins.

Csikszentmihalyi M (2003), Fluir en los negocios. Barcelona: airó.

Csikszentmihalyi M (2013), Fluir (Flow): Una Psicología de la Felicidad, España: KAIROS.

Csikszentmihalyi M, LeFevre J (1989), Optimal Experience in Work and Leisure. Committee on Human Development, University of Chicago. Journal of Personality and Social Psychology, Vol. 56, No. 5,815-822

Diener E, Biswas-Diener R (2008), Rethinking happiness: The science of psychological wealth. Malden: Blackwell Publishing.

Feris T, Castro M (2006), La importancia de la Satisfacción laboral y el clima organizacional para un buen desempeño en la organización (Diplomado, Universidad de La Sabana, Chía, Colombia),

Fernandez $M$, Martín L, Pérez $M$, González H (2011), Evaluación del flujo psicológico: Estudio comparativo entre dos escalas de medida. Revista de Psicología y Educación, 10(1), 75-96.

Figueiredo, Alberola, Gil-Monte, García-Juesas (2012) Síndrome de quemarse por el trabajo y satisfacción laboral en profesionales de enfermería

Forbes R (2013), La psicología organizacional positiva y la mejora organizacional. Éxito Empresarial, 227, 1-3.

Fullagar C, Mills M (2008), Motivation and flow: Toward an understanding of the dynamics of the relation in architecture students. The Journal of
Psychology, 142 (5), 533-553.

Gable S, Haidt J (2005), What (and why) is positive psychology? Review of General Psychology, 9 (2), 102-110.

García-Calvo T, Jiménez R, SantosRosa F, Reina R, Cervelló E (2008), Psychometric properties of the Spanish version of the Flow State Scale. Spanish journal of psychology, 11(2), 660-669.

García D (2010), Satisfacción Laboral. Una aproximación teórica. Contribuciones a las Ciencias Sociales: Universidad de Málaga, España. Extraído de http://www.eumed.net/rev/ cccss/09/dgv.htm.

Granados J, Salazar M, Gómez J (2011), Clima organizacional, satisfacción laboral y su relación con el desempeño laboral en trabajadores de una PYME de servicios de ingeniería. Universidad del Magdalena.

Han S (1988), The relationship between life satisfaction and flow in elderly Korean immigrants. In M. Csikszentmihalyi \& I. S. Csikszentmihalyi (Eds.), Optimal experience: Psychological studies of flow in consciousness (pp. 138-149), New York: Cambridge University Press.

Ishimura I, Kodama M (2006), Dimensions of flow experience in Japanese college students: Relation between flow experience and mental health. Journal of Health Psychology, 13, 23-34.

Jackson S, Marsh H (1996), Development and validation of a scale to measure optimal experience: The Flow State Scale. Journal of Sport and Exercise Psychology; 18:17-35.

Leibovich de Figueroa N, Maglio A (2012), La experiencia del fluir (Flow) en médicos argentinos. Instituto de Investigaciones, Facultad de Psi- 
Motivación, satisfacción laboral y estado de flow en los trabajadores de la salud Pizarro Ruz, Jenny; Fredes Collarte, Dailet; Inostroza Peña, Carolina y

Torreblanca Urbina. Elisa

cología, Universidad de Buenos Aires - Consejo Nacional de Investigaciones Científicas y Técnicas (CONICET), Argentina.

López Mas J (2005),Motivación laboral y gestión de recursos humanos en la teoría de Frederick Herzberg. Gestión en el Tercer Milenio, 8(15), 25-36.

Luthans F (2002), The need for and meaning of positive organizational behavior. Journal of Organizational Behavior, 23 (6), 695-706.

Lyubomirsky S, King L, Diener E (2005), The benefits of frequent positive affect: Does happiness lead to success? Psychological Bulletin, 131(6), 803-855.

Mañas M, Salvador C, Boada J, González E, Agulló E (2007), La satisfacción y el bienestar psicológico como antecedentes del compromiso organizacional. Psicothema, 19(3),395-400.

Mesurado B (2008), Validez Factorial y Fiabilidad del Cuestionario de Experiencia Óptima (Flow) para niños y adolescentes. Argentina.

Millán A, García-Álvarez D, D’Aubeterre M (2014), Efecto de la inteligencia emocional y flujo de trabajo sobre estresores y bienestar psicológico: análisis de ruta en docentes. Revista Colombiana de Psicología, 23(1), 207-228.

Nader M, Peña S, Santa-Bárbara E (2014), Predicción de la satisfacción y el bienestar en el trabajo: hacia un modelo de organización saludable en Colombia. Estudios Gerenciales, 30(130), 31-39.

Navarro J, Quijano S (2003), Dinámica no lineal en la motivación en el trabajo: propuesta de un modelo y resultados preliminares. Psicothema, 15 (4), 643-649.
Orta A, Sicilia A (2015) Investigando los momentos óptimos en el deporte:

Una revisión del constructo flow. Revista Brasileña de Ciencia del Deporte, 37(1), 96-103.

Ospina, García, Moliner, Molina, Martínez-Tur, (2012), Relaciones entre ruptura del contrato psicológico y burnout: un estudio en trabajadores de atención a personas con discapacidad intelectual.

Pearce J, Ainley M, Howard S (2005), The ebb and flow of online learning. Computers in Human Behavior, 21(5), 745-771.

Pecino $V$, Mañas $M$, Díaz $P$, López J, Llopis J (2015), Clima y satisfacción laboral en el contexto universitario.

Pérez J, Fidalgo M (2007), NTP: 394: Satisfacción laboral: escala general de satisfacción. (Informe NTP: 394), Instituto Nacional de Seguridad e Higiene en el Trabajo.

Peterson C, Park N, Seligman M (2005), Orientations to happiness and life satisfaction: The full life versus the empty life. Journal of Happiness Studies, 6, 25-41.

Price J, Mueller C (1986), Absenteeism and turnover among hospital Employees. Greenwich, CT: JAIPress.

Quijano S, Navarro J (1998), Un modelo integrado de la motivación en el trabajo: Conceptualización y medida. Revista de Psicología del Trabajo y de las Organizaciones, 14, 193 216.

Ripoll P, Caballer A, Martínez Tur V, García E, Peiró J (1988), Antecedentes y consecuencias del cambio de nivel jerárquico en el trabajo. $R e$ vista Latinoamericana de Psicología, 30(2), 203-222.

Rodríguez A, Cifre E, Salanova $M$, 
Aborg C (2008), Technoflow among Spanish and Swedish students: A Confirmatory Factor Multigroup Analysis. Anales de Psicología, 24, 42-48.

Salanova M, Martínez I, Cifré E, Schaufeli W (2005) ¿Se pueden vivir experiencias óptimas en el trabajo? Analizando el flow en contextos laborales. Rev. De Psi. GralyAplic, 58(1), 89-100.

Salanova M, Bakker A, Llorens S (2006), Flow at work: Evidence for an upward spiral of personal and organizational resources. Journal of Happiness Studies, 7, 1-22.

Salanova M (2008), Organizaciones saludables y desarrollo de recursos humanos. Estudios financieros. Revista de trabajo y seguridad social: Comentarios, casos prácticos: recursos humanos, (303), 179-214.

Santander X, Tordesilla C (2005), Salud mental en trabajadores de Salud Primaria.

Schui G, Krampen G (2010), Bibliometric analyses on the emergence and present growth of positive psychology. Applied Psychology: Health and wellbeing, 2(1), 52-64.

Seligman M, Csikszentmihalyi M (2000), Positive psychology: An introduction, American Psychologist, 55, 5-14.

Smith J (2005), Flow theory and GIS: is there aconnection for learning? International. Researchin
Geographical and Environmental Education4 (3), 223-230.

Steers R, Mowday R, Shapiro D (2004), The future of work motivation theory. Academy of Management review, 29 (3), 379-387.

Sheldon K, King L (2001), Why Positive Psychology is Necessary. American Psychologist, 56 (3), 216-7.

Tejada D (2013), Lo que es la atención primaria de la salud: algunas consideraciones a casi treinta y cinco años de Alma- Ata.

Tomasina (2012) Los problemas en el mundo del trabajo y su impacto en salud. Crisis financiera actual.

Walter L, Gallegos A, Velarde O (2013) Satisfacción Laboral en Trabajadores de Dos Tiendas por Departamento: Un Estudio Comparativo

Warr, Cook, Wall (1979), Positive organizational behavior: an idea who seetime has truly come. Journal of Organizational, 24,437-442.

Wise R (1987), Sensorimotor modulation and the variable action pattern (VAP): Toward a noncircular definition of drive and motivation, Psychobiology, 15 (1), 7-20.

Wilpert B (1995), Organizational Behavior. Annual Review of Psychology, 4, 59-90.

Zubiri F (2013), Satisfacción y motivación profesional. Canales del Sistema Sanitario de Navarra, 36 (2), 193196.

- $\quad$ Esta obra está bajo una licencia de Creative Commons Reconocimiento-NoComercial- Compartirlgual 3.0 Unported. http://creativecommons.org/licenses/by-nc-sa/3.0/deed.es_ES 